\title{
Gegenseitige Umsetzung von Semicarbazonen und Phenylhydrazonen
}

von

\section{Dr. Gustav Knöpfer.}

Aus dem chemischen Laboratorium der Landes-Oberrealschule in Brünn.

Mit Unterstiitzung der Gesellschaft zur Fördernng deutscher Kunst, Wissenschaft und Literatur in Böhmen.

(Vorgelegt in der Sitzung am 20. Jänner 1910.)

Zur Frage der gegenseitigen Verdrängbarkeit von Stickstoffradikalen, die an Kohlenstoff doppelt gebunden sind, habe ich in meiner letzten Arbeit ${ }^{1}$ durch die Überführung einer Reihe von Azinen in Hydrazone einen Beitrag geliefert und hierbei auf anderweitig beobachtete ähnliche Verdrängungserscheinungen hingewiesen, denen ich zunächst noch einige weitere Fälle aus der Literatur anfüge.

Georg Pinkus ${ }^{2}$ setzte das Methylglyoxalbenzoylosazon durch Erhitzen mit Phenylhydrazin für sich in das Phenylosazon um. Auwers und Richter ${ }^{3}$ haben das $l$-Arabinosephenylhydrazon und Oxim durch Behandeln mit der entsprechenden Base in das $p$-Bromphenylosazon übergeführt. Eibner und Hoffmann ${ }^{4}$ verdrängten den Anilinrest im Anilchinophtalon durch den Phenylhydrazinrest. Ofner ${ }^{5}$ hat in seinen Untersuchungen über Methyl- und Benzylphenylhydrazone der Zuckerarten gezeigt, daß der sekundäre Hydrazinrest durch

1 Monatshefte für Chemie, 30, 804 (1908).

2 Berl. Ber., 31, 35 (1898).

3 Berl. Ber., 32, 3387 (1899).

1 Berl. Ber., 37, 3019 (1904).

5 Berl. Ber., 37, 3362 (1904). 
den Phenylhydrazinrest vertretbar ist. Ähnlich haben auch Votoček und Voudraček ${ }^{1}$ nachgewiesen, da $\beta$ man aus dem Benzal-Methylphenylhydrazon und Benzal-p-Nitrophenylhydrazon durch Einwirkung von Phenylhydrazin das Phenylhydrazon erhalten könne, und daß dieses Verhalten auch für die Hydrazone und Osazone der Zuckerarten Gültigkeit habe. Wilhelm Meister ${ }^{2}$ schließt aus dem Eintritt des Hydrazinrestes an Stelle von $\mathrm{N}, \mathrm{H}$ und $\mathrm{O}$ in der Methazonsäure auf das Vorhandensein einer Oximgruppe in dieser Säure. Schließlich hat H. Bilt $z^{3}$ beobachtet, daß die Oximgruppe im Diacetylmonoxim und Benzoinoxim durch den Rest des Harnstoffes substituiert werden kann. In weiterer Verfolgung dieses Themas habe ich nun die gegenseitige Umsetzung der Semicarbazone und Hydrazone untersucht, worüber ich nachstehend berichte.

Die Umsetzungen gelangen nach beiden Richtungen nahe$\mathrm{zu}$ in allen untersuchten Fällen. Die Reaktion ist umkehrbar und verläuft naturgemäß nie vollständig. Es tritt vielmehr ein Gleichgewichtszustand ein, der in erster Reihe durch das Mengenverhältnis der einwirkenden Massen und nur in untergeordnetem Maße von der Temperatur abhängt. Es hat sich ergeben, daß bei der Einwirkung von maximal 5 Molekülen des zersetzenden Reagens die Umsetzung in der Regel praktisch vollständig eintrat.

Da auf diesem Wege ein Einblick in die relative Größe der Bindungsfestigkeit der beiden in Betracht kommenden Atomgruppen nicht $\mathrm{zu}$ gewinnen war, habe ich dieses Ziel dadurch $z u$ erreichen versucht, daß ich auch das Verhalten der Aldehyde, resptive Ketone auf ein molekuläres Gemenge von Phenylhydrazin und Semicarbazid prüfte. Allein auch hier bildete sich entweder ein Gemenge der möglichen Verbindungen oder ließ die Bevorzugung der Bildung von nur einer keine Regelmäßigkeit erkennen. Auf keinen Fall war die Löslichkeit der entstandenen Verbindung für deren Entstehen bestimmend, da die Semicarbazone durchwegs schwer lösliche, die Hydrazone leicht lösliche Körper sind.

1 Berl. Ber., 37, 3845 (1904).

2 Berl. Ber., 40, 3436 (1907).

3 Berl. Ber., 41, 1884 (1908). 
Schließlich habe ich der Analogie halber auch die bei den Azinen beobachtete Reaktion auf ihre Umkehrbarkeit geprüft und untersucht, ob sich Hydrazone in Azine umsetzen lassen. Die Verdrängbarkeit der Azingruppe durch den Phenylhydrazinrest vollzog sich dort ausschließlich als Folge verschiedener Affinitätsgröße der bejden Radikale und daraus war zu schließen, daß diese Reaktion nicht auch im umgekehrten Sinne durchführbar sein werde. Die Versuche, deren Ergebnisse ich an dieser Stelle einfüge, bestätigten diese Annahme.

Die Phenylhydrazone folgender Aldehyde, respektive Ketone wurden in dieser Richtung untersucht.

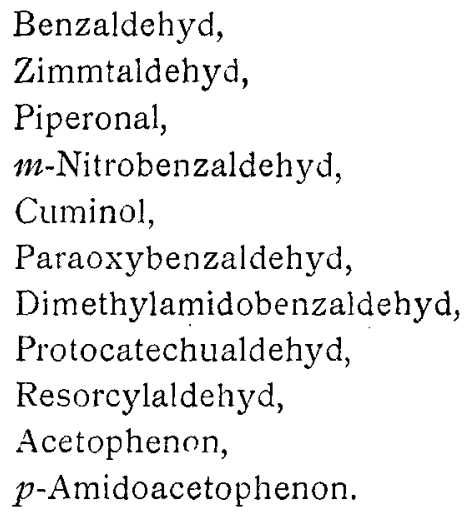

Die Hydrazone wurden in Alkohol gelöst, mit der wässerigen Lösung eines etwa $z w e i f a c h e n$ Überschusses der theoretischen Menge von Hydrazinsulfat und Soda versetzt und längere Zeit sich selbst überlassen. Hierauf wurde mit Wasser versetzt und die Fällung untersucht.

Bloß beim Resorcylaldehyd hat sich das Azin gebildet, in allen übrigen Fällen ${ }^{1}$ trat, wie zu erwarten war, die Umsetzung nicht ein.

Die Umsetzung der Semicarbazone in die Phenylhydrazone erfolgte durchaus in der Hitze und entweder in

1 Beim Versetzen der alkoholischen Lösung des p-Amidoacetophenonhydrazons mit der wässerigen Lösung des Hydrazinsulfats schieden sich nach einiger Zeit Krystalle des schwefelsauren Salzes des Hydrazons aus. Es ist, wie das salzsaure Salz in Wasser schwer löslich und schmilzt bei $215^{\circ}$ unter Zersetzung. Aus dem Hydrazon direkt hergestelltes Sulfat erwies sich damit identisch. 
alkoholischer oder eisessigsaurer Lösung. Im letzteren Falle wurde stets vorher die Beständigkeit des umzusetzenden Semicarbazons gegen Eisessig geprüft. $\mathrm{Zu}$ der Lösung des Semicarbazons wurde 1 Molekül Phenylhydrazin gefügt und erst, wenn diese Menge nicht ausreichte, ein Überschuß der Base zugesetzt.

Die entgegengesetzte Reaktion, die Überführung der Phenylhydrazone in die Semicarbazone (in der Folge als Umkehrung « bezeichnet), wurde unter womöglich gleichen. Lösungsverhältnissen wie die Umsetzung ausgeführt und zu diesem Zwecke das Phenylhydrazon in Alkohol gelöst und hierzu die erforderliche Menge von salzsaurem Semicarbazid und Kaliumacetat, in möglichst wenig Wasser gelöst, gefügt.

Die sich hier abspielenden Verdrängungserscheinungen werden durch folgende Gleichung ausgedrückt:

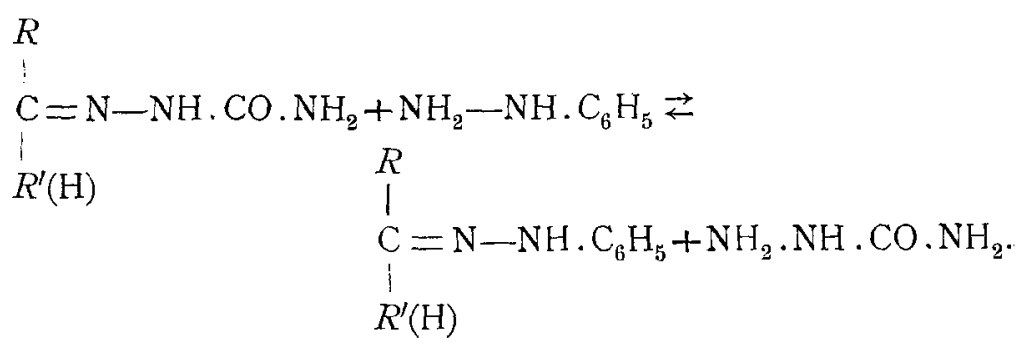

\section{Benzaldehyd.}

Das aus Benzaldehyd und salzsaurem Semicarbazid in wässeriger Lösung erhattene Semicarbaz on setzte sich schon bei der Einwirkung molekularer Mengen von Phenylhydrazin bei 18 stündigem Kochen in alkoholischer Lösung fast vollständig in das Hydrazon um. Das Reaktionsprodukt, im Vakuum eingeengt, schied Krystalle vom Schmelzpunkt des Hydrazons, $156^{\circ} 1$ aus. In Eisessig erfolgt die Umsetzung schon nach kurzem Kochen.

Umkehrung. In die alkoholische Lösung des Hydrazons wurde die wässerige Lösung von salzsaurem Semicarbazid und

1 Annalen, 190, 134 (1877), E. Fischer. 
Kaliumacetat gegossen. Es zeigte sich, daß die Reaktion sowohl in der Kälte als auch in der Hitze stattfand, und daß die Umsetzung bei Einwirkung von 3 Molekülen Semicarbazid nahezu vollständig erfolgt. Das Semicarbazon, mit Wasser gefällt und aus Alkohol umkrystallisiert, schmilzt bei 214 bis $216^{\circ}$.

Aldehyd + Phenylhydrazin + Semicarbazid. Zu der alkoholischen Lösung des Phenylhydrazins (1 Molekül) wurde die konzentrierte wässerige Lösung von saizsaurem Semicarbazid (1 Molekül) und Kaliumacetat hinzugefügt und die alkoholische Lösung des Benzaldehyds (1 Molekül) zugesetzt. Die vollkommen klare Mischung wurde in zwei Teile geteilt, wovon der eine zwei Stunden erhitzt, der andere einen Tag stehen gelassen wurde. Die Aufarbeitung der Reaktionsprodukte ergab, daß sich in beiden Fällen bloß das Hydrazon gebildet hatte, zumindest entzog sich etwa entstandenes Semicarbazon der Agnoszierung. In weiteren analog ausgeführten Versuchen wurde die Menge des Semicarbazids um je ein Molekül gesteigert und es ergab sich, daß für diesen Aldehyd bei Anwesenheit von 1 Molekül Phenylhydrazin 6 Moleküle Semicarbazid erforderlich waren, um zur Semicarbazonbildung zu führen. Hingegen machte sich ein wesentlicher Einfluß der Reaktionstemperatur nicht bemerkbar.

\section{Salicylaldehyd.}

Die Komponenten (Semicarbazon und Phenylhydrazin) wurden in alkoholischer Lösung 10 Stunden gekocht, wobei sich die Lösung klärte und eine grünliche Farbe annahm. Beim Eindunsten schieden sich Krystalle des Hydrazons, Schmelzpunkt $142^{\circ}, 2$ aus. Die Umsetzung erfolgte erst bei Einwirkung von 2 Molekülen Phenylhydrazin. In Eisessig fand sie schon nach kurzem Aufkochen statt, doch tritt da vorerst die Bildung des Azins ein, ${ }^{3}$ welches sich dann weiter ins Hydrazon umsetzte. Dał3 jedoch bei der Umsetzung in alkoholischer Lösung

1 Annalen, 270, 34 (1892), Thiele.

2 Berl. Ber., 17, 575 (1884), E. Fischer.

3 Berl. Ber., 31, 4299 (1901), W. Borsche. 
eine intermediäre Bildung von Azin nicht vor sich gehe, wurde durch einen gesonderten Versuch festgestelit. Bei zehnstündigem Kochen des Semicarbazons in Alkohol schied es sich wieder unverändert rein weiß aus. Eine auch nur teilweise vor sich gegangene Azinbildung würde sich durch Gelbfärbung bemerkbar gemacht haben.

Umkehrung. Molekulare Gemenge des Hydrazons und salzsaurem Semicarbazid wurden in verdünnter alkoholischer Lösung sich selbst überlassen. Mit Wasser gefällt, scheiden sich Krystalle aus, die mit Alkohol gereinigt, den Schmelzpunkt $229^{\circ}$ zeigen. In der Hitze erfolgt die Verdrängung ebenfalls.

Aldehyd + Phenylhydrazin + Semicarbazid. Verfährt man in gleicher Weise wie beim vorigen Aldehyd, so zeigt sich, daß in der Hitze fast nur das Hydrazon, beim längeren Kochen hingegen ein Gemisch von diesem und Semicarbazon entsteht.

\section{3. $p$-Oxybenzaldehyd.}

Das Semicarbazon entsteht aus dem Aldehyd und salzsaurem Semicarbazid in wässeriger Lösung und stellt eine vollkonmen weiße, bei $218^{\circ}$ schmelzende Krystallmasse dar, während Borsche und Boltzer ${ }^{2}$ es als gelbliche, bei 223 bis $225^{\circ}$ schmelzende Verbindung beschrieben. Die Umsetzung ins Hydrazon gelingt in alkoholischer Lösung nicht, hingegen erfolgt sie in 20 prozentiger Essigsäure. Das Semicarbazon wurde durch Aufkochen in genannter Säure gelöst und etwa 4 Moleküle Phenylhydrazin zugesetzt. Es schied sich alsbald eine voluminöse Fällung aus, die den Schmelzpunkt des Hydrazons, $178^{\circ},{ }^{3}$ zeigte. Das Semicarbazon für sich in gleicher Weise mit 20 prozentiger Essigsäure behandelt, blieb vollständig unverändert. Auch hier würde sich eine auch nur partielle Bildung von Azin durch Gelbfärbung der Lösung kenntlich gemacht haben.

I Berl. Ber., 34, 4299 (1901), W. Bors che.

2 Berl. Ber., 34, 2098 (1901).

3 Annalen, 248, 99 (1889), Rudolph. 
Umkehrung. Hydrazon, salzsaures Semicarbazid (4 Moleküle) und die entsprechende Menge Kaliumacetat wurden in verdünnter alkoholischer Lösung zur Einwirkung gebracht und die Lösung nach mehreren Stunden mit Wasser versetzt. Die entstandene Fällung ergab mit Alkohol gereinigt Semicarbazon. Bej der Einwirkung von bloß 1 Molekül Semicarbazid trat die Verdrängung nicht ein.

Aldehyd +Phenylhydrazin +Semicarbazid. Sowohl in der Kälte wie auch bei einstündigem Kochen hat sich in diesem Falle nur das Hydrazon gebildet.

\section{Vanillin.}

In alkoholischer Lösung konnte das Semicarbazon nicht ins Hydrazon umgesetzt werden, es trat Verharzung ein. Bei Anwendung von Eisessig, gegen den das Semicarbazon beständig ist, wird dieser Übelstand zwar nicht ganz vermieden, doch konnten aus der Reaktionsmasse Krystalle ausgeschieden werden, die an ihrem Schmelzpunkt und der leichten Löslichkeit in Alkohol als das Hydrazon zu erkennen waren. Schmelzpunkt des Hydrazons $105^{\circ} .1$

Umkehrung. Molekulare Mengen reagierten weder in der Kälte noch in der Hitze. Erst bei der Einwirkung von 4 Molekülen Semicarbazid erfolgte die Bildung des Semicarbazons. Schmelzpunkt $229^{\circ} .^{2}$

Aldehyd + Phenylhydrazin + Semicarbazid. Aus der alkoholischen Lösung scheiden sich nach mehreren Stunden Krystalle aus. Die gleiche Verbindung entsteht beim Fällen der Mutterlauge mit Wasser. Es hat sich das Semicarbazon gebildet. In der Hitze verläuft die Reaktion in gleicher Weise.

\section{Anisaldehyd.}

Auch bei dem Semicarbazon dieses Aldehyds gelang die Umsetzung ins Hydrazon nur in eisessigsaurer Lösung und bei Einwirkung von 4 Molekülen Phenylhydrazin. Schmelzpunkt des Hydrazons $121^{\circ} .^{3}$

1 Berl. Ber., 18, 1662 (1885), Tiemann und Kees.

2 Monatshefte für Chemie, 26, 344 (1905), Ott.

3 Annalen, 248, 103 (1888), Rudolph. 
Umkehrung. Molekulare Mengen wurden in verdünnter alkoholischer Lösung aufeinander wirken gelassen. Es entsteht sogleich eine schwache Fällung. Die Flüssigkeit wurde geschüttelt und in zwei Teile geteilt. Die eine Hälfte ergab nach einstündigem Kochen unverändertes Hydrazon. Die andere Hälfte wurde bei gewöhnlicher Temperatur beobachtet, und zwar wurde wiederum ein Teil sogleich aufgearbeitet und liefert ebenfalls unverändertes Hydrazon, während der andere Teil einen Tag sich selbst überlassen wurde. Nach dieser Zeit wurde mit Wasser verdünnt. Die entstandene Fällung ist nunmehr in Alkohol sehr schwer löslich und zeigt den Schmelzpunkt des Semicarbazons 203 bis $204^{\circ} .1$

Aldehyd + Phenylhydrazin + Semicarbazid. Sowohl in der Kälte wie in der Hitze bildete sich ein Gemenge des Semicarbazons und Hydrazons.

\section{Cuminol.}

Die Umsetzung erfolgte bei fünfstündigem Kochen des Semicarbazons mit 4 Molekülen Phenylhydrazin in Eisessig, gegen welchen jedoch das Semicarbazon nicht völlig resistent ist. Bei mehrstündigem Kochen des Semicarbazons mit Eisessig scheidet sich wohl die Hauptmasse des Semicarbazons wieder unverändert aus, aber die Mutterlauge zeigt deutlich den Geruch des Cuminols. Schmelzpunkt des Hydrazons $129^{\circ} .{ }^{2}$

Umkehrung. Das Hydrazon wurde in heißem Alkohol gelöst und die Lösung der molekularen Menge von salzsaurem Semicarbazid in sehr wenig Wasser hinzugefügt. Nach 2 Stunden schieden sich seidenglänzende Krystalle des Semicarbazons aus, die bei $207^{\circ}$ schmelzen, entgegen $202^{\circ} 3$ der Literaturangabe. In der Hitze verläuft der Vorgang in gleicher Weise.

Aldehyd + Phenylhydrazin + Semicarbazid. Aus der verdünnten alkoholischen Lösung schieden sich nach einiger Zeit geringe Mengen Hydrazon aus. Die Hauptmasse der

1 Journal für prakt. Chemie (2), 68,247 , Wah $1 \mathrm{~b}$ a um.

2 Annalen, 248, 101 (1888), Rudolph.

3 Journal für prakt. Chemie (2), 66,55 , Wahl baum und $\mathrm{Huthig.}$ 
Reaktion verblieb in Lösung, aus der sie mit Wasser gefällt wurde. Der Schmelzpunkt der Fällung weist auf das Semicarbazon, das sich demnach vornehmlich gebildet hat.

\section{Piperonal.}

Erst bei zehnstündigem Kochen der alkoholischen Lösung von 1 Molekül Semicarbazon und 6 Molekülen Phenylhydrazin trat die Umsetzung ein. Es resultiert das bei 102 bis $103^{\circ}$ schmelzende Hydrazon. ${ }^{1}$

Umkehrung. Berechnete Mengen der Komponenten in der üblichen Weise aufeinander einwirkend, scheiden bald schöne Krystalle des Semicarbazons aus. Schmelzpunkt 230 bis $233^{\circ} .^{2}$

Aldehyd + Phenylhydrazin + Semicarbazid. Aus der Lösung schieden sich bald reichliche Mengen von Krystallen aus. Dieselbe Verbindung lieferte auch die Mutterlauge nach dem Fällen mit Wasser und zeigt den Schmelzpunkt des Semicarbazons. Hier hat sich demnach blob diese Verbindung gebildet.

\section{Furfurol.}

Das Semicarbazon entsteht leicht in wässeriger Lösung und bildet bräunlichgelbe Nadeln, die in Alkohol leicht, schwerer

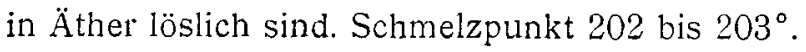

Die Analyse dieser noch nicht bekannten Verbindung ergab:

$0.1247 \mathrm{~g}^{\mathrm{S}}$ Substanz lieferten $30.1 \mathrm{~cm}^{3}$ feuchten Stickstoff, $b=740 \mathrm{~mm}, t=14^{\circ}$.

In 100 Teilen:

$$
\text { N } \ldots \ldots \ldots \ldots \underbrace{\text { Gefunden }}_{27 \cdot 59} \quad \underbrace{\begin{array}{c}
\text { Berechnet für } \\
\mathrm{C}_{6} \mathrm{H}_{7} \mathrm{O}_{2} \mathrm{~N}_{3}
\end{array}}_{27 \cdot 4 \overline{5}}
$$

In Alkohol erfolgt keine Umsetzung. Nach vierstündigem Kochen entstand beim Zusatz von Wasser auch nach längerem Stehen keine Fällung. In Eisessig tritt Hydrazonbildung ein,

4 Annalen, 248, 103 (1888), Rud olph.

5 Monatshefte fü: Chemie, 26. 345 (1905), Ott. 
doch geht ihr, wie der Versuch ergibt, eine Spaltung des Semicarbazons voraus.

Umkehrung. Salzsaurer Semicarbazid wirkt auf das Hydrazon dieses Aldehyds nicht ein. Daß jedoch die Bildung des Semicarbazons in alkoholischer Lösung möglich sei, wurde dadurch bewiesen, daß der Aldehyd in viel Alkohol gelöst und 1 Molekül salzsaures Semicarbazid - ohne Kaliumacetat in einigen Tropfen Wasser gelöst zugesetzt wurde. Die Bildung des Semicarbazons ging glatt von statten.

Aldehyd + Phenylhydrazin + Semicarbazid. Molekulare Mengen in der wiederholt angegebenen Weise aufeinander wirkend, lieferten das Hydrazon. Schmelzpunkt 97 bis $98^{\circ} .^{1}$

\section{9. o-Nitrobenzaldehyd.}

Das Semicarbazon fällt sogleich beim Vereinigen der Aldehydlösung mit der wässerigen Lösung von salzsaurem Semicarbazid und Kaliumacetat als gelbes, in Alkohol kaum lösliches Krystallpulver aus, das bei $242^{\circ}$ (statt bei $256^{\circ}{ }^{2}$ ) unter Zersetzung schmilzt. Mit Phenylhydrazin in alkoholischer Lösung trat selbst bei zehnstündigem Kochen die Umsetzung nicht ein. Dagegen führte die Verwendung von Eisessig als Lösungsmittel, gegen welches das Semicarbazid resistent ist, zur Bildung des Hydrazons. Es war durch die hyazinthrote Farbe und den Schmelzpunkt 150 bis $153^{\circ}{ }^{3}$ leicht zu erkennen.

Ich habe auch versucht, auf das Semicarbazon Phenylhydrazin für sich ohne jedes Lösungsmittel einwirken zu lassen. Nach kurzem Kochen, während welchem starke Gasentwicklung $\mathrm{zu}$ beobachten ist, wird die Flüssigkeit klar und hell und erstarrt nach dem Erkalten zu einem Krystallbrei, der nicht das erwartete Hydrazon, sondern eine erst bei $222^{\circ}$ schmelzende Verbindung vorstellt.

1 Berl. Ber., 17, 574 (1884), E. Fis cher.

2 Anralen, 283, 25 (1894), Thiele und Stange.

3 Annalen, 232, 232 (1885), Pickel. 
Die Analyse dieser Verbindung ergab:

I. $0.1485 \mathrm{~g}$ Substanz lieferten $27.4 \mathrm{~cm}^{3}$ feuchten Stickstoff. $t=24^{\circ}$, $b=740 \mathrm{~mm}$.

II. $0.1503 \mathrm{~g}$ Substanz lieferten $26.0 \mathrm{~cm}^{3}$ feuchten Stickstoff. $t=17^{\circ}$, $b=752 \mathrm{~mm}$.

In 100 Teilen:

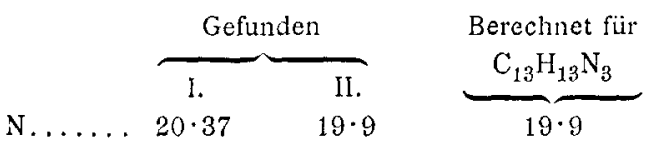

Das Phenylhydrazin hat auf die Nitrogruppe des Aldehyds reduzierend eingewirkt und zur Bildung von $o$-A mid obenzolphenylhydrazon geführt, das von Eliasberg und Friedländer ${ }^{1}$ direkt erhalten wurde. Dieselbe Verbindung in vorzüglicher Ausbeute erhielt ich auch bei kurzem Sieden des Aldehyds in Phenylhydrazin. Hier konnte ich beide Phasen der Einwirkung genau unterscheiden. Nach kurzem Erhitzen bilden sich die roten Krystalle des Hydrazons vom Schmelzpunkt 150 bis $153^{\circ}$ und bei fortgesetztem Kochen wird die Reaktionsmasse hell und ergibt das bei $222^{\circ}$ schmelzende o-Amidobenzolphenylhydrazon.

Anfangs war ich der Meinung, daß sich aus dem Phenylhydrazin durch teilweise Oxydation Anilin gebildet und daß dieses auf das Semicarbazon unter Bildung eines Phenylsemicarbazons eingewirkt haben könnte. Letztere Reaktion drückt sich durch die Gleichung

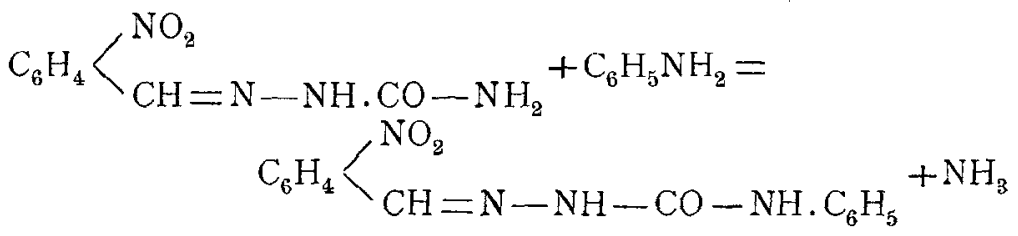

aus und ist von Borsche ${ }^{2}$ an einer Reihe von Semicarbazonen ausgeführt worden. Da auch die Analysenresultate für diese

1 Berl. Ber., 25, 1752 (1892).

2 Berl. Ber, 34, 4297 (1901) und 37, 3177 (1904). 
Annahme sprachen, habe ich die vermutete Verbindung direkt hergestellt und zu diesem Behufe o-Nitrobenzalsemicarbazon einige Minuten mit Anilin gekocht. Ich erhielt nun einen von dem früher beschriebenen durchaus verschiedenen Körper, gelbe, bei $195^{\circ}$ schmelzende Krystalle, die zweifellos das $o-N$ itrobenzalphenylsemicarbazon vorstellen.

Die Analyse bestätigte diese Annahme; sie ergab:

$0 \cdot 1556 \mathrm{~g}$ Substanz lieferte $27.4 \mathrm{~cm}^{3}$ feuchten Stickstoff, $t=16^{\circ}, b=744 \mathrm{~mm}$.

In 100 Teilen:

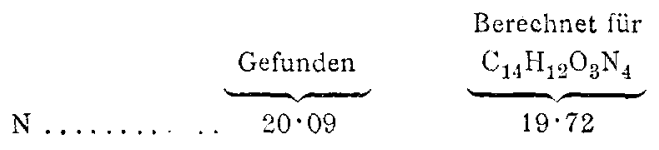

Umkehrung. 1 Molekül Hydrazon in verdünnter alkoholischer Lösung mit 4 Molekülen salzsaurem Semicarbazid und Kaliumacetat zusammengebracht, gaben sowohl nach vierstündigem Sieden als auch bei längerem Stehen in der Kälte unverändertes Hydrazon.

Aldehyd + Phenylhydrazin + Semicarbazid. Bald nach der Vereinigung der Substanzen in verdünnter alkoholischer Lösung tritt bereits in der Kälte reichliche Krystallausscheidung von gebildetem Hydrazon ein und auch die Mutterlauge führte nach der Aufarbeitung zu derselben Verbindung:

\section{0. $m$-Nitrobenzaldehyd.}

Die Umsetzung des Semicarbazons in das Hydrazon vollzog sich bloß in Eisessig, das Semicarbazon allein wird durch diesen nicht verändert. Das Hydrazon bildet die charakteristischen roten Krystalle vom Schmelzpunkt $121^{\circ}$.

Umkehrung. In der Kälte tritt die Reaktion auch hier nicht ein. Läßt man jedoch auf das Hydrazon in der Hitze 2 Moleküle salzsaures Semicarbazid und die entsprechende Menge Kaliumacetat einwirken, so findet nach dreistündigem Kochen die Verdrängung statt. Nach dem Versetzen mit Wasser und Umkrystallisieren der entstandenen Fällung aus Alkohol 
erhält man das bei $246^{\circ} 1$ schmelzende, schwach gelblich gefärbte Semicarbazon.

Aldehyd + Phenylhydrazin +Semicarbazid. Ausder verdünnten alkoholischen Lösung schieden sich in der Kälte nach einigem Stehen gelbrote Krystalle aus. Sie wurden mit Alkohol gekocht, worin sie sich zum Teil lösen. Der unlösliche Teil ist gelb und schmilzt bei $247^{\circ}$, es ist das Semicarbazon, während sich der lösliche Teil beim Verdunsten des Alkohols in gelbroten Krystallen ausscheidet und das Hydrazon darstellt.

\section{1. $p$-Nitrobenzaldehyd.}

Das Semicarbazon verhielt sich den vorerwähnten Isomeren durchaus gleich. Es ging bei der Behandlung mit Phenylhydrazin glatt in das Hydrazon vom Schmelzpunkt $155^{\circ} 2$ über.

Umkehrung. Unter gleichen Umständen wie bei der $o$-Verbindung bleibt das Hydrazon in der Kälte und auch bei mehrstündigem Erhitzen von Semicarbazid unangegriffen.

Aldehyd + Phenylhydrazin + Semicarbazid. Es bildet sich bloß das Hydrazon und scheidet sich zum Teil schon nach einigem Stehen aus der Lösung aus.

\section{Zimmtaldehyd.}

In Alkohol tritt die Umsetzung nicht ein, hingegen erfolgt sie bereits bei Anwendung eines Moleküles Phenylhydrazin in Eisessig. Nach kurzem Aufkochen bildet sich das Hydrazon. Schmelzpunkt $168^{\circ} .^{3}$

Umkehrung. Äquimolekulare Mengen von Zimmtaldehydphenylhydrazon in Alkohol gelöst und salzsaures Semicarbazid, in möglichst wenigWasser gelöst, zusammengebracht, scheiden zunächst unverändertes Hydrazon aus. Es wurde durch Erwärmen Lösung bewirkt. Nach weiterem Zusatz von

\footnotetext{
1 Annalen, 283, 25, Thiele und Stange.

2 Annalen, 232, 232 (1885), Rudolph.

3 Berl. Ber., 17, 575 (1884), E. Fischer.
} 
Alkohol wurde die klare Lösung sich selbst überlassen und nach einem Tage mit Wasser gefällt. Nach entsprechender Reinigung schmolz die Verbindung bei $214^{\circ}$, dem Schmelzpunkt des Semicarbazons. Literaturangabe 215 bis $216^{\circ} .^{1}$

\section{Dimethylaminobenzaldehyd.}

Darstellung des Semicarbazons. Zur wässerigen Lösung von salzsaurem Semicarbazid wurde die berechnete Menge des Aldehyds, in Alkohol gelöst, zugesetzt. Es scheiden sich hellrote Blättchen aus der tiefroten Flüssigkeit ab. Nach dem Umkrystallisieren aus Alkohol, worin sich die Verbindung schwer löst, erhält man weiße Nadeln, die sich wohl bei 221 bis $222^{\circ}$ zersetzen, bei $250^{\circ}$ aber erst schmelzen. Offenbar findet beim Erhitzen vorerst die Bildung des Azins statt, wie dies W. Borsche ${ }^{2}$ und auch schon Kipping ${ }^{3}$ bei anderen Semicarbazonen beobachtet haben. In der Tat schmilzt das hier in Betracht kommende Azin bei 250 bis $253^{\circ} .^{4}$

Der Verlauf der Zersetzung wird durch die Gleichung ausgedrückt:

$$
\begin{aligned}
& 2 \mathrm{C}_{6} \mathrm{H}_{4} \backslash \begin{array}{l}
\mathrm{N}\left(\mathrm{CH}_{3}\right)_{2} \\
\mathrm{CH}=\mathrm{N} . \mathrm{NH} . \mathrm{CO} \cdot \mathrm{NH}_{2}
\end{array}=\mathrm{C}_{6} \mathrm{H}_{4}\left\langle\begin{array}{l}
\mathrm{N}\left(\mathrm{CH}_{3}\right)_{2} \\
\mathrm{CH}=\mathrm{N}
\end{array}\right. \\
& \mathrm{C}_{6} \mathrm{H}_{4}\left\langle\begin{array}{l}
\mathrm{CH}=\mathrm{N} \\
\mathrm{N}\left(\mathrm{CH}_{3}\right)_{2}
\end{array}\right. \\
& +\mathrm{NH}_{2} \cdot \mathrm{CO} \cdot \mathrm{NH} \cdot \mathrm{NH} . \mathrm{CO} \cdot \mathrm{NH}_{2} \text {. }
\end{aligned}
$$

Neben dem Azin entsteht Hydrazodicarbonamid, respektive dessen Zersetzungsprodukte.

Die Analyse des bis jetzt unbekannten Semicarbazons ergab:

1 Journ. of the chem. Soc., 77,230, Young und Witham.

2 Berl. Ber., 34, 4297 (1901).

3 Chem. Zentralbl. 1900, I., 762.

4 Berl. Ber., 39, 808 (1906). 
$0.1937 \mathrm{~g}$ Substanz lieferte $45 \cdot 5 \mathrm{~cm}^{3}$ feuchten Stickstoff. $t=13^{\circ}, b=738 \mathrm{~mm}$.

In 100 Teilen:

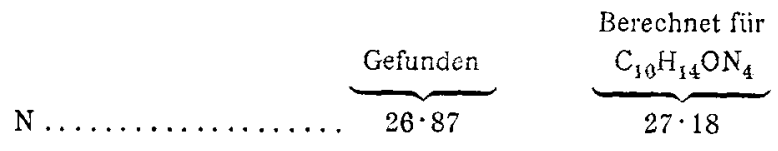

Behufs Umsetzung in das Hydrazon wurde das Semicarbazon in alkoholischer Lösung mit 3 Molekülen Phenylhydrazin versetzt und 3 Stunden gekocht. Es resultiert das bei $148^{\circ}{ }^{1}$ schmelzende Hydrazon.

Umkehrung. Diese vollzog sich nur dann vollständig; wenn der etwa dreifache Überschuß von salzsaurem Semicarbazid zur Einwirkung gelangte. Das Semicarbazon scheidet sich als weiße, krystallinische Masse, doch nur zum Teil aus; die Hauptmasse fällt erst nach dem Zusatz von Soda.

Aldehyd +Phenylhydrazin + Semicarbazid. Ein Teil des Gemenges wurde in der Kälte sich selbst überlassen. Er erstart alsbald und die abgesaugten Krystalle erweisen sich als das Hydrazon. Den gleichen Körper liefert in geringen Mengen auch die Mutterlauge nach dem Fällen mit Wasser.

Der andere Teil wurde 3 Stunden gekocht und hierauf sogleich mit Wasser gefällt. Auch hier entstand bloß das Hydrazon.

\section{Resorcylaldehyd.}

Das bis jetzt unbekannte Semicarbazon dieses Aldehyds bildet sich leicht, wenn man zur wässerigen Lösung von salzsaurem Semicarbazid die berechnete Menge des Aldehyds, in Alkohol gelöst, hinzufügt und das Gemenge schüttelt. Schon nach kurzer Zeit scheiden sich hellgelbe Krystalle des Semicarbazons aus. Die Verbindung ist in sehr geringen Mengen in Alkohol und auch in siedendem Wasser löslich, leichter in Essigsäure, aus deren Lösung $(50 \%)$ sie umkrystallisiert wurde. Der Schmelzpunkt konnte nicht ermittelt werden. Bei $210^{\circ}$ wird die Substanz rot und bei $260^{\circ}$ bereits ganz dunkel, ohne

1 Berl. Ber., 20, 3195 (1887), Knoefler und Boessneck. 
sich jedoch verflüssigt $\mathrm{zu}$ haben. Auch hier dürfte die Umwandlung in das bei $310^{\circ}$ noch nicht schmelzende Azin ${ }^{1}$ vor sich gehen.

Ich habe trotz aller Bemühungen, die sich sowohl auf die Reinigung der Substanz wie auf verschiedentliche Modifikationen in der Analyse bezogen, keine gut stimmenden Analysenresultate erzielen können, trotzdem die Darstellung dieser Verbindung, sowie auch ihre Bildung aus dem Hydrazon kaum ihre Identität bezweifeln lassen. Ich bin zu der Annahme genötigt, daß beim Umkrystallisieren dieser Verbindung eine, wenn auch nur geringe Azinbildung vor sich geht, denn das Azin verlangt $10 \cdot 3$, das Semicarbazon $21.5 \%$ N. Es folgen einige der ausgeführten Analysen.

I. $0.2204 \mathrm{~g}$ Substanz lieferten $37 \cdot 3 \mathrm{~cm}^{3}$ feuchten Stickstoff. $t=17^{\circ}$, $b=750 \mathrm{~mm}$.

II. $0.1702 \mathrm{~g}$ Substanz lieferten $31.1 \mathrm{~cm}^{3}$ feuchten Stickstoff. $t=14^{\circ}$, $b=751 \mathrm{~mm}$.

1II. $0.1767 \mathrm{~g}$ Substanz lieferten $31.9 \mathrm{~cm}^{3}$ feuchten Stickstoff. $t=20^{\circ}$, $b=736 \mathrm{~mm}$.

IV. $0.1141 \mathrm{~g}$ Substanz lieferten $20.9 \mathrm{~cm}^{3}$ feuchten Stickstoff. $t=20^{\circ}$, $b=735 \mathrm{~mm}$.

V. $0 \cdot 1978 g$ Substanz lieferten $36.6 \mathrm{~cm}^{3}$ feuchten Stickstoff. $t=20^{\circ}$ $b=727 \mathrm{~mm}$.

In 100 Teilen:

\begin{tabular}{|c|c|c|c|c|}
\hline & \multicolumn{3}{|c|}{ Gefunden } & \multirow{2}{*}{$\begin{array}{l}\text { Berechnet für } \\
\mathrm{C}_{8} \mathrm{H}_{9} \mathrm{O}_{3} \mathrm{~N}_{3}\end{array}$} \\
\hline & II & IV & V & \\
\hline$\ldots \ldots \ldots \ldots \ldots$ & $21 \cdot 2$ & $20 \cdot 1$ & $20 \cdot 3$ & $21 \cdot 53$ \\
\hline
\end{tabular}

Ins Hydrazon ließ sich das Semicarbazon beim Kochen mit 2 Molekülen Phenylhydrazin in alkoholischer Lösung umsetzen. Nach etwa $1 \frac{1}{2}$ Stunden trat Lösung und damit Bildung des Hydrazons, Schmelzpunkt $159^{\circ},{ }^{2}$ ein. In Eisessig geht die Umsetzung bereits nach kurzem Aufkochen vor sich.

Umkehrung. Ein Teil der Lösung wurde in der Kälte sich selbst überlassen und ergab nach der Fällung mit Wasser ein bei $215^{\circ}$ schmelzendes Produkt, offenbar ein Gemenge von Hydrazon und Semicarbazon. Der zweite Teil wurde gekocht

1 Monatshefte für Chemie, 30,35 (1909), Knöpfer.

2 Annalen, 248, 105 (1888), Rudolph. 
und lieferte ein um einige Grade höher schmelzendes Produkt, als es das Hydrazon verlangt. Auch hier ist demnach ein Gemenge der beiden möglichen Verbindungen, vorherrschend jedoch Hydrazon, entstanden.

Aldehyd + Phenylhydrazin + Semicarbazid. Beim Stehen der Lösung scheiden sich die in Alkohol sehr schwer löslichen, hochschmelzenden Krystalle des Semicarbazons aus. Das Filtrat ergab nach dem Fällen mit Wasser das bei $159^{\circ}$ schmelzende Hydrazon. Es haben sich demnach beide Verbindungen gebildet.

\section{Protocatechualdehyd.}

Betreffs der Darstellung und der Eigenschaften des bis jetzt ebenfalls unbekannten Semicarbazons gilt das gleiche wie für die vorangehende Verbindung. Auch sie zeigt keinen eigentlichen Schmelzpunkt, sondern beginnt sich von $230^{\circ}$ an unter Dunkelfärbung zu zersetzen. Der Reinigung und Analyse setzt sie die gleichen Schwierigkeiten wie ihr Isomeres entgegen.

Die Analysen ergaben:

I. $0.1883 \mathrm{~g}$ Substanz lieferten $35.6 \mathrm{~cm}^{3}$ feuchten Stickstoff. $t=23^{\circ}$, $b=732 \mathrm{~mm}$.

II. $0 \cdot 1810 \mathrm{~g}$ Substanz lieferten $32 \cdot 8 \mathrm{~cm}^{3}$ feuchten Stickstoff. $t=16^{\circ}$. $b=740 \mathrm{~mm}$.

III. $0.2041 \mathrm{~g}$ Substanz lieferten $38.0 \mathrm{~cm}^{3}$ feuchten Stickstoff. $t=20^{\circ}$, $b=734 \mathrm{~mm}$.

IV. $0.1479 \mathrm{~g}$ Substanz lieferten $28.5 \mathrm{~cm}^{3}$ feuchten Stickstoff. $t=22^{\circ}$, $b=735 \mathrm{~mm}$.

In 100 Teilen:

\begin{tabular}{|c|c|c|c|c|c|}
\hline & \multicolumn{4}{|c|}{ Gefunden } & \multirow{2}{*}{$\begin{array}{r}\begin{array}{c}\text { Berechnet für } \\
\mathrm{C}_{8} \mathrm{H}_{9} \mathrm{O}_{3} \mathrm{~N}_{3}\end{array} \\
\end{array}$} \\
\hline & I & II & III & IV & \\
\hline & $20 \cdot 7$ & $20 \cdot 6$ & $20 \cdot 6$ & $21 \cdot 28$ & $21 \cdot 53$ \\
\hline
\end{tabular}

Die Überführung in Hydrazon, Schmelzpunkt $176^{\circ},{ }^{1}$ vollzieht sich genau unter denselben Bedingungen wie beim vorigen Semicarbazon. Die Umsetzung erfolgt erst bei der Einwirkung von 4 Molekülen Phenylhydrazin.

1 Monatshefte für Chemie, 17, 245 (1886), Wegscheider. 
Umkehrung. Die anfangs klare Lösung vom Hydrazon und salzsaurem Semicarbazid (4 Moleküle) in verdünntem Alkohol scheidet nach längerem Stehen bei gewöhnlicher Temperatur das Semicarbazon aus.

Aldehyd + Phenylhydrazin + Semicarbazid. Beim Stehen schied sich keine Fällung aus und auch beim Verdünnen mit Wasser entsteht nur eine schwache, harzige, in Alkohol leicht lösliche Ausscheidung, aus der sich keine bestimmte Verbindung isolieren ließ.

\section{Acetophenon.}

Das Semicarbazon wurde von W. Borsche ${ }^{1}$ erhalten und der Schmelzpunkt mit 198 bis $199^{\circ}$ angegeben. Ich erhielt die Verbindung durch Zusatz der alkoholischen Lösung des Ketons zur wässerigen Lösung von salzsaurem Semicarbazid und sie schmolz bei $203^{\circ}$.

Ins Hydrazon konnte ich das Semicarbazon nicht umsetzen; in Alkohol blieb die Reaktion aus und Eisessig wirkt zersetzend.

Umkehrung. Bei Einhaltung des wiederholt angegebenen Verfahrens setzt sich das Hydrazon unter der Einwirkung von 3 bis 4 Molekülen Semicarbazid in der Kälte in das Semicarbazon um.

Keton + Phenylhydrazin + Semicarbazid. In der Kälte schied die verdünnte alkoholische Lösung keine Fällung aus. Ein Teil der Lösung wurde mit Wasser verdünnt und lieferte nach entsprechender Aufarbeitung das Semicarbazon. Der andere Teil wurde zwei Stunden gekocht und gab beim Versetzen mit Wasser eine nur geringe Ausscheidung, die nicht weiter untersucht wurde.

\section{7. $p$-Amidoacetophenon.}

Das Semicarbazon wird erhalten, wenn man die alkoholische Lösung des Ketons zu einer verdünnten Lösung von salzsaurem Semicarbazid und Kaliumacetat hinzufügt und längere Zeit stehen läßt. Eine geringe Menge chlorhältiger

1. Berl. Ber., 34, 4301 (1901). 
Krystalle scheidet sich aus, es ist das salzsaure Salz des Semicarbazons. Aus diesem sowie aus der Mutterlauge wird das Semicarbazon durch Kochen mit Soda in Freiheit gesetzt. Es bildet nach dem Umkrystallisieren aus Alkohol, worin es sich in der Hitze löst, gelbliche, bei $250^{\circ}$ unter Zersetzung schmelzende Krystalle.

Die Analyse der bisher nicht bekannten Verbindung ergab:

$0.1220 \mathrm{~g}$ Substanz lieferten $31.4 \mathrm{~cm}^{3}$ feuchten Stickstoff. $t=20^{\circ}, b=748 \mathrm{~mm}$.

In 100 Teilen:

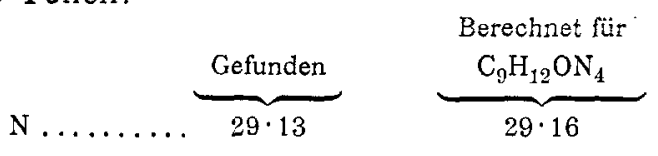

Umsetzung ins Hydrazon. Das Hydrazon dieses Ketons wurde bisher im freien Zustande nicht erhalten, sondern ist nur als salzsaures Salz ${ }^{1}$ bekannt.

In meiner vorigen Arbeit habe ich bereits angeführt, daß man durch Kochen des salzsauren Hydrazons mit Sodalösung das Hydrazon in Gestalt eines flockigen Niederschlages wohl erhält, daß es jedoch nicht gelingt, diese Verbindung durch Umkrystallisieren zu reinigen, da hierbei stets Verharzung eintritt. Auch die direkte Behandlung des Ketons mit Phenylhydrazin in alkoholischer Lösung führte nicht zum gewünschten Ziele.

Von sehr günstigem Erfolg war hingegen folgendes Verfahren begleitet. $1 \mathrm{~g}$ Keton wurde in etwa $30 \mathrm{~cm}^{3} 50$ prozentiger Essigsäure gelöst und mit $2 \mathrm{~g}$ frisch destilliertem Phenylhydrazin versetzt. Die hellgelbe Mischung wurde bei gewöhnlicher Temperatur stehen gelassen. Nach 2 Stunden schied sich das Hydrazon in schönen, großen Krystallen von fast weißer Farbe ab. Sie sind bereits rein. Aus verdünntem Alkohol lassen sie sich nunmehr umkrystallisieren, wobei aber ein beträchtlicher Teil verloren geht, ohne daß die Reinheit der Substanz erhöht würde. Es resultieren $0.5 \mathrm{~g} \mathrm{Hydrazon}$; harte, schwach gelbliche Krystalle, die bei 110 bis $114^{\circ}$ schmelzen.

1 Berl. Ber., 20, 512 (1887), Münchmeyer. 
Die Analyse ergab:

I. $0.1838 \mathrm{~g}$ Substanz lieferten $30.3 \mathrm{~cm}^{3}$ feuchten Stickstoff. $t=18^{\circ}$, $b=745 \mathrm{~mm}$.

II. $0 \cdot 1963 \mathrm{~g}$ Substanz lieferten $33.2 \mathrm{~cm}^{3}$ feuchten Stickstoff. $t=24^{\circ}$, $b=744 \mathrm{~mm}$.

In 100 Teilen:

N ........ $\overbrace{\begin{array}{c}\text { I. } \\ 18 \cdot 71\end{array} \begin{array}{c}\text { II. } \\ 18.78\end{array}}^{\text {Gefunden }} \underbrace{\begin{array}{c}\text { Berechnet für } \\ \mathrm{C}_{14} \mathrm{H}_{12} \mathrm{~N}_{3}\end{array}}_{18.66}$

In alkoholischer Lösung mit Salzsäure behandelt, scheidet sich das salzsaure Salz des Hydrazons aus. Es ist nunmehr rein weiß und zersetzt sich erst bei $215^{\circ}$, während die gleiche Verbindung von Münchmeyer als ein rotes bei $207^{\circ}$ schmelzendes Salz beschrieben wird.

Das Semicarbazon dieses Ketons ließ sich in alkoholischer Lösung weder mit freiem noch mit salzsaurem Phenylhydrazin in das Hydrazon, respektive dessen Salz umsetzen, es biieb selbst bei andauernder Einwirkung in der Hitze unverändert. Ehe die Umsetzung in Essigsäure vorgenommen wurde, mußte das Verhalten des Semicarbazons zu dieser Säure untersucht werden. Beim Kochen mit Eisessig erhält man eine klare Lösung, die nach dem Zusatz von Wasser und auch beim Kochen mit Soda keine Trübung zeigt. Erst nach 24 Stunden scheidet sich das Semicarbazon wieder aus. Es hat vermutlich erst eine Spaltung und hierauf die Rückbildung des Semicarbazons stattgefunden. In 50 prozentiger Essigsäure hingegen kann das Semicarbazon ohne Zersetzung gelöst werden, es fällt daraus durch Kaliumhydroxyd sofort wieder unverändert aus. In einer solchen Lösung wurde Phenylhydrazin auf das Semicarbazon in der Kälte einwirken gelassen. Nach einem Tage wurde mit Kaliumhydroxyd gefällt. Es entstand die rötlich gefärbte Verbindung des Hydrazons, die behufs Agnoszierung in das salzsaure Salz überführt wurde. In der Tat erhält man das charakteristische, in Wasser schwer lösliche Salz, das bei $209^{\circ}$ unter Zersetzung schmilzt.

Umkehrung. Diese Reaktion verlief sowohl in alkoholischer wie auch in essigsaurer Lösung negativ. 


\section{8. $\omega$-Bromacetophenon.}

Mit salzsaurem Semicarbazid und Kaliumacetat reagiert das Keton in normaler Weise. Das entstandene Semicarbaz on stellt weiße, in Alkohol leicht lösliche Krystalle dar, die bei $146^{\circ}$ schmelzen. Die Analyse dieser bisher unbekannten Verbindung ergab:

I. $0.1825 \mathrm{~g}$ Substanz lieferten $27.0 \mathrm{~cm}^{3}$ feuchten Stickstoff. $i=16^{\circ}$, $b=747 \mathrm{~mm}$.

II. $0 \cdot 1542 \S$ Substanz lieferten $23.4 \mathrm{~cm}^{3}$ feuchten Stickstoff. $t=20^{\circ}$, $b=739 \mathrm{~mm}$.

III. $0 \cdot 1810 \mathrm{~g}$ Substanz gaben $0 \cdot 1340 \mathrm{~g} \mathrm{Ag} \mathrm{Br}$.

In 100 Teilen:

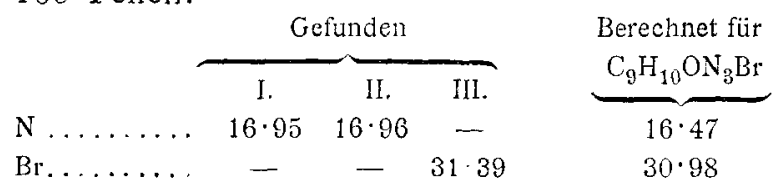

Über die Einwirkung von Phenylhydrazin auf $\omega$-Bromacetophenon liegen Untersuchungen von $\mathrm{O}$. $\mathrm{He} \mathrm{B}^{1}$ und J. $\mathrm{Cu} 1-$ man ${ }^{2}{ }^{2}$ vor. Es bildet sich demzufolge nicht das Hydrazon sondern unter Austritt von Bromwasserstoff eine Verbindung, welcher nach $\mathrm{Culmann}$ die Formel

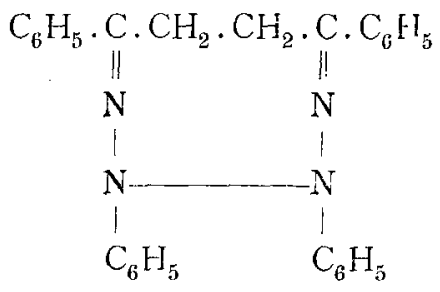

zukommt und die er als Tetraphenyltetracarbazon bezeichnet. Sie ist gelb und schmilzt bei $137^{\circ}$.

Bei meinen Versuchen über die Einwirkung von Phenylhydrazin auf das oben beschriebene Semicarbazon erhielt ich weder das Hydrazon noch den von den vorgenannten Autoren

1 Annalen, 232, 234 (1885).

2 Annalen, 258, 235 (1890). 
beschriebenen Körper, an dessen statt bildete sich vielmehr eine harzige, nicht weiter zu reinigende Masse.

\section{9. $\omega$-Chloracetophenon.}

Das Verhalten dieses Ketons war dem des vorigen völlig analog.

Das Semicarbarzon stellt schöne weiße, in Alkohol schwer lösliche Krystalle dar, die bei $156^{\circ}$ schmelzen.

Die bis jetzt unbekannte Verbindung ergab bei der Analyse:

I. $0.1418 \mathrm{~g}$ Substanz lieferten $24.9 \mathrm{~cm}^{3}$ feuchten Stickstoff. $t=14^{\circ}$, $b=736 \mathrm{~mm}$.

II. $0.2606 \mathrm{~g}$ Substanz lieferten $0.1743 \mathrm{~g} \mathrm{AgCl}$.

In 100 Teilen:

\begin{tabular}{|c|c|c|c|}
\hline & \multicolumn{2}{|c|}{ Gefunden } & \multirow{2}{*}{$\begin{array}{r}\begin{array}{r}\text { Berechnet für } \\
\mathrm{C}_{9} \mathrm{H}_{10} \mathrm{ON}_{3} \mathrm{Cl}\end{array} \\
\end{array}$} \\
\hline & I. & II. & \\
\hline $\mathrm{N} \ldots \ldots$ & $19 \cdot 96$ & - & $19 \cdot 85$ \\
\hline $\mathrm{Cl} \ldots \ldots$ : & - & $16 \cdot 55$ & $16 \cdot 78$ \\
\hline
\end{tabular}

Phenylhydrazin reagiert mit dem Keton in gleicher Weise wie mit der entsprechenden Bromverbindung, es entstand auch hier die bei $137^{\circ}$ schmelzende, halogenfreie gelbe Verbindung.

Die Einwirkung von Phenylhydrazin auf das Semicarbazon führte ebenfalls $z u$ keiner faßbaren Verbindung.

\section{Benzylidenaceton.}

Das Semicarbazon ist von Rupe und Schlochoff 1 dargestellt worden und bildet weiße, bei $185^{\circ}$ schmelzende Blättchen.

In alkoholischer Lösung tritt erst bei Einwirkung von 3 bis 4 Molekülen Phenylhydrazin in der Hitze nach 2 Stunden Hydrazon bildung ein. Das Hydrazon schmilzt bei $155^{\circ}$ gegen $157^{\circ}$, die E. Fischer beobachtete, und zeigt mit Salzsäure die charakteristische Gelbfärbung.

Umkehrung. Die verdünnte alkoholische Lösung des Hydrazons mit 4 Molekülen salzsaurem Semicarbazid versetzt,

1 Berl. Ber., 17, 576 (1884), E. Fis cher. 
scheidet nach 12 Stunden beim Fällen mit Wasser weiße Krystalle aus, die mit dem Semicarbazon identisch sind.

Keton + Phenylhydrazin + Semicarbazid. Bald ist in der verdünnten alkoholischen Lösung eine reichliche Krystallbildung wahrzunehmen. Dieselbe Verbindung ist auch in der Mutterlauge gelöst und fällt nach dem Zusatz von Wasser. Sie besitzt den Schmelzpunkt des Hydrazons und zeigt auch die für das Hydrazon charakteristische Gelbfärbung beim Behandeln mit konzentrierter Salzsäure.

Beigefügte Tabelle gibt eine Übersicht der erhaltenen Resultate, wobei die Pfeile über den Rubriken den Sinn der darüberstehenden Umsetzung, die Zeichen plus und minus den Erfolg derselben ausdrücken sollen. Auch ist das jeweilig verwendete Lösungsmittel angeführt, weil dies für die Beurteilung der Reaktion nicht unwesentlich ist. Die halogenisierten Acetophenone sind mit Rücksicht auf ihr abnormales Verhalten zu Phenylhydrazin in diese Zusammenstellung nicht aufgenommen.

\begin{tabular}{|c|c|c|c|c|}
\hline $\mathrm{Nr}$. & Aldehyd, Keton & \multicolumn{3}{|c|}{ Semicarbazon $\rightleftarrows$ Hydrazon } \\
\hline 1 & Benzaldehyd .......... & $+($ Alkohol $)$ & $+($ ver & rohol) \\
\hline 2 & Salicylaldehyd ........ & + & + & $»$ \\
\hline 3 & $p$-Oxybenzaldehyd...$\ldots$ & $+(20 \%$ Essigsäure $)$ & $+>$ & » \\
\hline 4 & Vanillin . & $+($ Eisessig $)$ & $+\infty$ & $\gg$ \\
\hline 5 & Anisaldehyd ..... & + & $+\gg$ & » \\
\hline 6 & Cuminol............. & + & + & $\gg$ \\
\hline 7 & Piperonal... & $+($ Alkohol $)$ & + & » \\
\hline 8 & Furfurol $\ldots \ldots \ldots \ldots \ldots$ & - & $--\quad \gg$ & 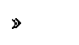 \\
\hline 9 & o-Nitrobenzaldehyd .. & $+($ Eisessig $)$ & $->$ & $»$ \\
\hline 10 & $m$-Nitrobenzaldehyd..... & + & $+>$ & $\gg$ \\
\hline 11 & $p$-Nitrobenzaldehyd $\ldots .$. & + & - & $\gg$ \\
\hline 12 & Zimmtaldehyd......... & + & + & » \\
\hline 13 & Dimethylamidobenzaldehyd & $+($ Alkohol $)$ & + & 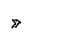 \\
\hline
\end{tabular}


G. Knöpfer, Umsetzung von Semicarbazonen.

\begin{tabular}{|c|c|c|c|c|}
\hline $\mathrm{Nr}$. & Aldehyd, Keton & \multicolumn{3}{|c|}{$\underset{\rightarrow}{\text { Semicarbazon }} \stackrel{\leftarrow}{\rightleftarrows}$ Hydrazon } \\
\hline 14 & Resorcylaldehyd.... & + (Alkohol) & $+($ ver & kohol) \\
\hline 15 & Protocatechualdehyd. & $+\quad$ & + & $»$ \\
\hline 16 & Acetophenon .......... & - & + & $\gg$ \\
\hline 17 & $p$-Amidoacetophenon ..... & $+(20 \%$ Essigsäure $)$ & - $\gg$ & » \\
\hline 18 & Benzylidenaceton. . & $+($ Alkohol $)$ & + & $\gg$ \\
\hline
\end{tabular}

